\title{
Coronary artery disease in periodontitis rat model
}

\author{
I Dewa Ayu Susilawati ${ }^{*}$, Suryono Suryono², Neira Najatus Sakinah ${ }^{3}$, \\ Maizirwan $\mathrm{Mel}^{4}$ \\ ${ }^{1}$ Department Biomedical Science, Faculty of Dentistry, \\ University of Jember, Indonesia \\ ${ }^{2}$ Department Cardiology Faculty of Medicine, University of Jember, \\ dr Soebandi Hospital, Jember. Indonesia \\ ${ }^{3}$ Department of Periodonsia, Faculty of Dentistry, University of Jember, Indonesia. \\ ${ }^{4}$ Bioprocess Engineering Research Group, Department of Biotechnology \\ Faculty of Engineering, International Islamic University Malaysia, Malaysia
}

\section{*Corresponding author: (I Dewa Ayu Susilawati) \\ Email : dewasusi@unej.ac.id}

\begin{abstract}
.
Context: Epidemiological and microbiological studies have reported association between periodontitis and coronary artery disease (CAD), experimental study that simulated cause-effect of periodontitis in CAD, however, was lacking. Aims: This study aimed to demonstrate the occurrence of coronary artery disease as a consequence of induced periodontitis in a rat model. Settings and Design: This in vivo experimental study using the post test only control group design. Methods and Material: A total of 12 rats (Rattus norvegicus) were divided into control and periodontitis groups (six rats in each group). Periodontitis was induced by injection of periodontitis bacteria Porphyromonas gingivalis in buccal gingival sulcus of left mandibular teeth, thrice a week for 4 wk. All rats were fed with normocholesterol diet. At the end of study, all rats were sacrificed. The rats'heart containing coronary arteries were removed, cut cross-sectionally and prepared for histochemistry assay. Statistical analysis used: Descriptive analysis was used to assess the frequency of CAD lesion, and $t$ test for IMT Results: All of rats in periodontitis group demonstrated signs of CAD. Coronary artery local inflammation indicated by leukocytes and erythrocyte accumulation were identified in all rats in periodontitis group $(100 \%)$. Parameters of intimal collagen disintegration and endotelial disintegration were also commonly occured (91.66 \% each), atheroma (41.66\%), stenosis $(41.66 \%)$. Periodontis group demonstrated significantly higher mean IMT ( $p<0.05$ ) compared to control group, $3.6 \mu \mathrm{m} \pm 1.4 \mu \mathrm{m}$ and $2.1 \mu \mathrm{m} \pm 0.7 \mu \mathrm{m}$, respectively. Conclusions: Periodontitis induced CAD. Periodontitis rat model might be used to represent as atherosclerotic model as well.
\end{abstract}

Keywords: Atherosclerotic model, in vivo, intima-media thickness, Porphyromonas gingivalis

How to cite this article: Susilawati et al. (2020): Coronary artery disease in periodontitis rat model, Ann Trop \& Public Health; S461 Vol. 23 Issue 3(A): 44-52.

DOI : http://doi.org/10.36295/ASRO.2020.23314 


\section{Introduction}

Interests in the role of chronic bacterial periodontitis (chronic bacterial inflammatory disease of tooth supporting tissue) in the pathogenesis of coronary artery disease (CAD) have been increasing during the last two decades. Studies have reported the association of periodontitis and increase risk of $\mathrm{CAD}^{[1],[2],[3] \cdot[4],[5],[6]}$. In addition, the main periodontitis bacteremia, Porphyromonas gingivalis (Coykendall, et al. 1980, Shah and Collins 1988) has been identified in specimen of coronary atherosclerotic plaque in patients who suffered from atherosclerotic cardiovascular disease ${ }^{[7],[8],[9]}$.

Periodontitis as a cause of CAD have been studied both in vitro and in vivo. Some studies have reported that periodontitis bacteremia were capable of inducing formation of foam cells and promoting the secretion of pro-inflammatory cytokines $^{[10],[11],[12]}$. This might subsequently lead to the development of atherosclerosis and acceleration of its early stage. $P$. gingivalis bacteremia have also been reported to induce coronary and aortic atherosclerosis in normocholesterolemic and hypercholesterolemic pigs ${ }^{[13]}$. To understand further the role of periodontitis in CAD, it is important to study using a periodontitis model, since it has the ability to delineate the pathway of infection from periodontal origin.

This study aimed to demonstrate the occurrence of coronary artery disease as a consequence of induced periodontitis in a rat model. Parameters of CAD were intima-media thickening, atheroma, stenosis, disintegration of endothelial cell and intimal collagen. In addition, local inflammatory signs i.e. leucocytes and erythrocyte accumulation in coronary arterial walls were studied as well.

\section{Materials and Methods}

\section{Animals and groups}

A number of $12( \pm 0.2 \mathrm{~kg}, 12$ wk old) male rats (Rattus norvegicus [Berkenhout, 1769]) in this research were purchased from the Department of Physiology Faculty of Medicine Brawijaya University, Malang, Indonesia. The animal were maintained under standard laboratory conditions in concordance with the guideline established by the institutional Animal Care and Ethics Committee No. 1100/H25.1.11/KE/2016. Animal treatment procedure was approved by Ethical committee at Faculty of Medicine, Universitas Jember, Indonesia. Dry pellet normocholesterol standard diet and water were given ad libitum. All rats were housed in pens in identical condition to minimize environment factors. A total of 12 rats were randomly divided into two groups: i) periodontitis, and ii) control (healthy, non-periodontitis). 


\section{Periodontitis rat model}

Periodontitis model was induced in rats by injecting periodontitis bacteremia $P$. gingivalis (ATTC, 33277) in buccal gingival sulcus of left mandibular teeth ${ }^{[14]}$. Before injection, these teeth were tided using a loop wire ligature $\left(\theta=5 \times 10^{-4} \mathrm{~m}\right)$, for obtaining a better retention for bacterial plaque that allowed bacterial accumulation on periodontal tissue leading to periodontitis. This procedure was performed under intramuscular anesthesia using ketamin $0.02 \mathrm{~mL} \mathrm{rat}^{-1}$. $P$. gingivalis was prepared in a culture medium Brain Heart Infusion Broth (BHI-B) enriched by vitamin $\mathrm{K}_{1}$ and Hemin (Sigma) under anaerobic atmosphere for $48 \mathrm{~h}$. To confirm bacterial purity, $P$. gingivalis was stained using Gram dye, and identification under light microscope shown that $P$. gingivalis was Gram negative bacteremia with uniform rod-shaped morphology. $P$. gingivalis concentration was adjusted to McFarland standard 0.5 . Chronic periodontitis was conditioned by regular injection of $5 \times 10^{-5} \mathrm{~L} P$. gingivalis thrice a week for $4 \mathrm{wk}$. Periodontitis was confirmed by the occurrence of alveolar bone resorption.

\section{Histologic samples preparation}

At the end of experiment, all of rats were fasted overnight then sacrificed using intramuscular ketamin $0.02 \mathrm{~mL} \mathrm{rat}^{-1}$. Their rats' heart containing coronary arteries were removed and fixed in $10 \%$ formalin (in [Phosphate Buffer Solution] PBS), and then trimmed crossectionally (perpendicular to the direction of blood flow) in about coronal half area of the heart, and prepared for histologic samples by frozen section. Serial cryosections $\left(1 \times 10^{-5} \mathrm{~m}\right.$ thickness $)$ were made and then mounted on to microscope slides (three sections on each slide).

\section{Histologic samples analysis}

To analyze coronary morphology and IMT, samples were stained using Hematoxylin-Eosin staning (Sigma), and collagen staining kit (Picrosirius Red, ScyTek, USA. The histologic images were assessed under light microscope magnification $\times 400$ and visualized using optilab microscope camera. Although samples were crossectional section of heart, however, analysis was focused on coronary artery only. Each sample was represented by two images of coronary artery, therefore, each group (consisted of six rats) represented by 12 images of coronary artery

\section{Analysis of coronary artery morphology and IMT}

Morphologic analysis was conducted to assess the CAD (atherosclerotic) indicators. There were the existence of atheroma (protruding of arterial wall into the lumen), stenosis/luminal narrowing/occlusion, these were analyzed qualitatively as present or absent. While coronary IMT was measured in the thickest area or atheroma. Measurement was done using available software in optilab $(\mu \mathrm{m})$.

\section{Analysis of intimal collagen thickness and integrity}

Intimal collagen thickness and integrity is focused since this structure composed a fibrous cap that is very important to maintain the resistance of the vessel and atherosclerotic plaque against rupture and thrombosis. Thinning and disintegration 
of intimal collagen could increase the risk of atherosclerotic plaque rupture. The present study analyzed the quality of intimal collagen as intact or disintegrated.

\section{Analysis of inflammatory markers}

To observe the occurence of local inflammation in coronary arteries, accumulation of leukocytes and erythrocytes on the arterial wall were observed.

\section{Statistic analysis}

Descriptive analysis was used to assess the frequency of CAD lesion parameters. Data of IMT were expressed as mean \pm standard deviation, and statistically analyzed by $\mathrm{t}$ test.

\section{Result}

All periodontitis rat models demonstrated signs of CAD. Morphometric analysis revealed that the periodontitis rat models had significantly thicker coronary artery IMT than control group $(p<0.05)$ (Table 1). Since IMT were measured in thickest area, the occurrence of atheroma seemed to contributed to the higher IMT mean in periodontitis group. Morphologic analysis revealed that signs of coronary atherosclerotic lesion in all samples of periodontitis group including atheroma, stenosis, endothelial denudation, intimal collagen thinning and disintegration (Table 2 and Figure 1). Signs of local inflammation was characterized by the accumulation of leukocytes and erythrocyte (Figure 2 and Figure 3). Atherosclerotic lesions were found in some specimens of control group as well, but in a lower frequency.

Table 1. Coronary artery intima-media thickness (IMT) of the rats

\begin{tabular}{lll}
\hline Group & $\mathrm{n}$ & $\begin{array}{l}\text { Intima-media thickness } \\
\text { Mean } \pm \mathrm{SD}\left(\times 10^{-6} \mathrm{~m}\right)\end{array}$ \\
\hline Control & 12 & $2.1 \pm 0.7$ \\
Periodontitis & 12 & $3.6 \pm 1.4$ \\
$P$ & \multicolumn{2}{l}{$0.003^{*}$} \\
\hline *significantly different, $p<0.05(\mathrm{t}$ test $)$ \\
$\mathrm{n}:$ number of specimen
\end{tabular}

Table 2. Coronary atherosclerotic lesion in periodontitis rat model

\begin{tabular}{lllll}
\hline \multirow{2}{*}{ Atherosclerotic lesion } & \multicolumn{2}{l}{ Control $(\mathrm{N}=12)$} & Periodontitis $\quad(\mathrm{N}=12)$ \\
\cline { 2 - 5 } & $\mathrm{n}$ & $(\%)$ & $\mathrm{n}$ & $(\%)$ \\
\hline Atheroma & 0 & 0 & 5 & 41.66 \\
Stenosis & 0 & 0 & 5 & 41.66 \\
Intimal collagen disintegration & 3 & 25 & 11 & 91.66 \\
Endotel disintegration & 5 & 41.66 & 11 & 91.66 \\
leukocytes \& erythrocyte & 2 & 16.66 & 12 & 100 \\
accumulation & & & & \\
\hline
\end{tabular}

$\mathrm{n} \quad$ : number of specimen

$\mathrm{N}$ : total number of specimens 

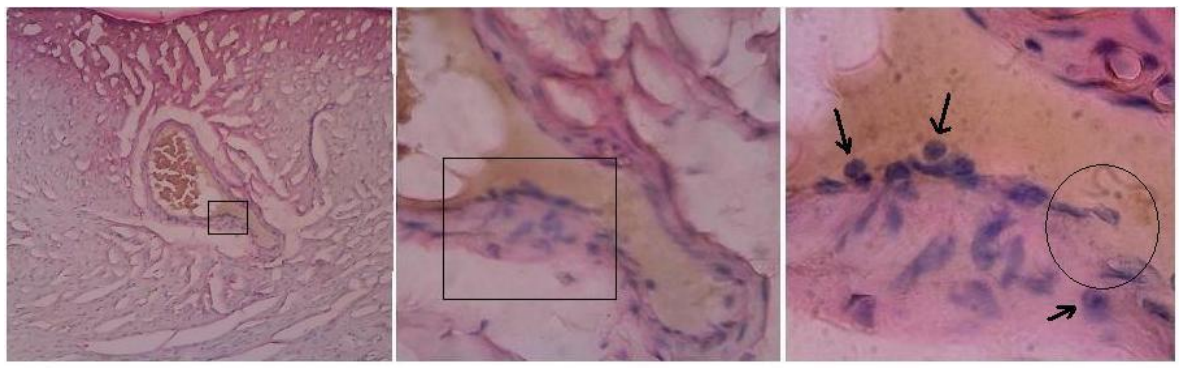

Figure 1. Coronary atherosclerosis in periodontitis rat model (Hematoxilin-Eosin staining). A, magnification $\times 100$ showed morphology atheroma (box) and luminal narrowing, $B$, magnification $\times 400$. C, magnification $\times 1000$ showed leukocytes infiltration (arrow), endothelial denudation (circle)
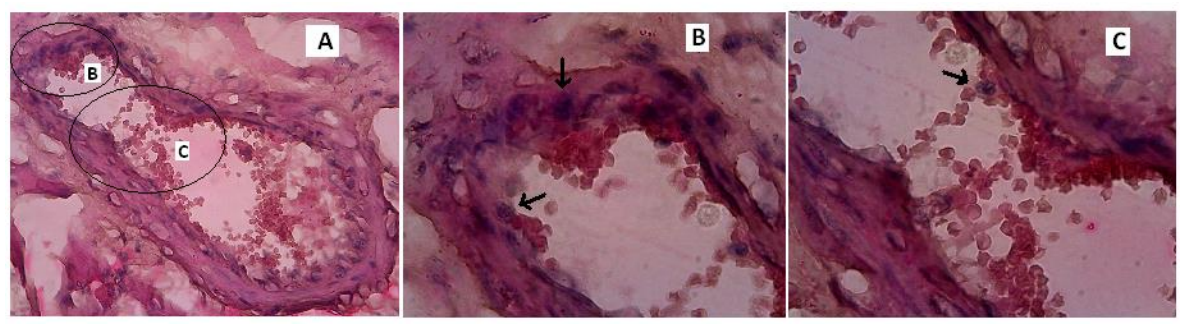

Figure 2. Coronary atherosclerosis in periodontitis rat model (Hematoxilin-Eosin staining). A, magnification $\times 400$ showed atheroma morphology (circles). B and C, magnification $\times 1000$ showed leucocyte infiltration (arrow) and erythrocyte accumulation in area atherosclerotic lesion
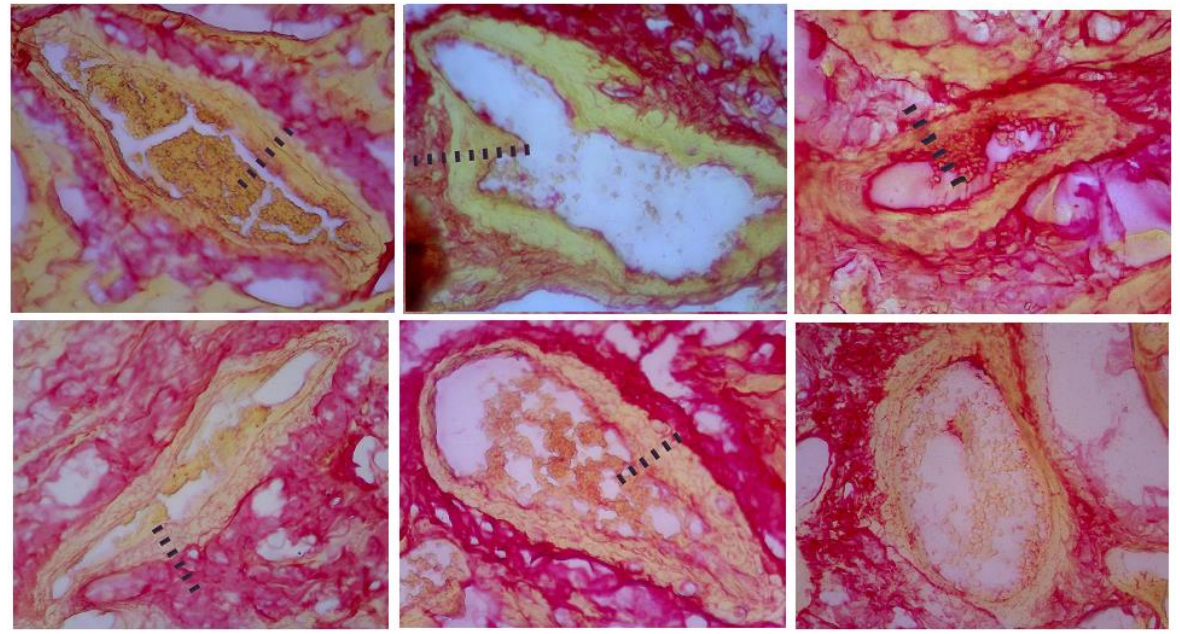

Figure 3. Worsening of coronary artery morphology in periodontitis rat model (Picro sirius red staining), indicated by arterial wall intimal thickening, progression of atheroma, stenosis and intimal collagen disintegration 


\section{Discussion}

Coronary artery disease (CAD) or Coronary atherosclerosis is an inflammatory disease characterized by thickening of coronary arterial wall, which is the result of accumulation of atheromatous plaques. This plaque is made up of fat mainly cholesterol (in the core) and cover by collagenous tissue called fibrous cap. The currently accepted hypothesis is that atherosclerosis develops as a response to injury and that is primarily a chronic inflammatory condition ${ }^{[15] \cdot[16],[17]}$. Bacterial inflammatory disease such as periodontitis had been alleged to play an important role in pathogenesis of atherosclerosis.

This study revealed the cause-effect of periodontitis in pathogenesis of CAD. Rat model that was created by $P$. gingivalis challenged on periodontal tissue mimicking periodontitis, and it was shown that periodontitis induced the formation of the coronary atherosclerotic lesion, characterized by thickening of the arterial wall, formation of atheroma, intimal collagen disintegration, endothelial denudation, and local inflammation is used in this research. These characters of the lesion were consistent with atherosclerosis.

This study is used periodontitis rat model in order to simulate the effect of infection from periodontal origin. Previously Li et al. demonstrated that mice with ApoEdeficient exhibited increased aortic atherosclerosis when challenged intravenously with invasive strain of $P$. gingivalis ${ }^{[11],[18]}$. Other study by Brodala et al. reported recurrent intravenous injection with $P$. gingivalis, mimicking periodontitisassociated bacteremia, promoted coronary artery and aortic atherosclerosis in pigs $^{[12],[13]}$. This study is supported and extended these finding. One important difference between this study and others previous studies was the challenged of $P$. gingivalis via periodontitis model, this method probably served evidence more closely to the real condition.

In this study, rats in both of periodontitis and non-periodontitis group were fed with the same normocholesterol standard diet, in order to exclude factor of lipid intake, and therefore we assumed that the independent variable was periodontitis. In other word, the distinctive feature of coronary artery structure was assumed due to periodontitis condition. Regarding the level of cholesterol, this study measured serum lipoprotein, low density lipoprotein (LDL) and high density lipoprotein (HDL), (data not shown). Periodontitis rat model exhibited higher serum LDL (although not significant, $p>0.05)$, and a significant lower HDL $(p<0.05)$. This can be noticed that periodontitis might affect atherogenesis indirectly via lipid metabolism. Further studies, however, were needed to confirm this.

Limitations noticed, this study did not measure the severity of periodontitis and level of bacteremia, therefore it could not be concluded, whether severity of periodontitis affected severity of atherosclerosis. Further studies however were needed to confirm the effect of duration and severity of periodontitis in pathogenesis atherosclerosis. 
Atherosclerosis is a chronic inflammatory disease, the dynamic structural change in arterial wall was alleged to be related to the dynamic change of systemic and vascular inflammatory condition. This study identified local vascular change of coronary artery due to the occurence of local inflammation, indicated by erythrocyte and leucocyte accumulation around atherosclerotic lesion. Local inflammatory response in coronary arterial wall probably due to circulating antigen from periodontitis origin.

\section{Acknowledgement}

This study was funded by grant (No. 0481/UN25.3.1/LT/2017) from Ministry of Research Technology and High Education, Republic Indonesia. Special thanks to Laboratory staffs of Biomedical Science Department of Faculty Dentistry, Jember University, Indonesia, Mrs. Wahyu, Mr. Agus and Mr. Pinardi for helping us with the laboratory work.

\section{References}

[1] Beukers NGFM, van der Heijden GJMG, Van Wijk AJ, Loos BG. Periodontitis is an independent risk indicator for atherosclerotic cardiovascular diseases among 60174 participants in a large dental school in the Netherland. J Epidemiol Community Health. 2017;71(1):37-42. https://jech.bmj.com/content/71/1/37.short

[2] Bokhari SAH, Khan AA, Leung WK, and Wajid G. Association of periodontal and cardiovascular diseases: South-Asian studies 2001-2012. J Indian Soc Periodontol. 2015;19(5):495-500. https://pdfs.semanticscholar.org/fbca/b96d59ffdba55e8fe0f5146d0f8f3bf9086d.pdf ? ga $=2.83168927 .862756258 .1562122639-1800790154.1562122639$

[3] Joshipura K, Zevallos JC, and Ritchie CS. Strength of evidence relating periodontal disease and atherosclerotic disease. Compend Contin Educ Dent. 2009;30(7): 430-439.

https://www.researchgate.net/publication/26815522_Strength_of_Evidence_Relatin g_Periodontal_Disease_and_Atherosclerotic_Disease

[4] Seymour GJ, Ford PJ, Cullinan MP, Leishman S, Yamazaki K. Relationship between periodontal infections and systemic disease. Clinical Microbiology and Infection. 2007;13:3-10.

https://www.sciencedirect.com/science/article/pii/S1198743X14624599

[5] Latronico M, Segantini A, Cavallini F, Mascolo A, Garbarino F, Bondanza S, Debbia EA, Blasi G. Periodontal disease and coronary heart disease: An epidemiological and microbiological study. New Microbiol.2007;30(3):221-8. https://pdfs.semanticscholar.org/1a8c/a317e4a5000767cc872a70235e3257a0f85d.p $\underline{\mathrm{df}}$

[6] Geerts SO, Legrand V, Charpentier J, Albert A, Rompen EH. Further evidence of the association between periodontal conditions and coronary artery disease. J Periodontol. 2004;75(9):1274-80. https://aap.onlinelibrary.wiley.com/doi/abs/10.1902/jop.2004.75.9.1274 
[7] Haraszthy VI, Zambon JJ, Trevisan M, Zeid M, Genco RJ. Identification of periodontal pathogens in atheromathous plaques. J. Periodontol. 2000; 71:1554-60. https://www.researchgate.net/publication/12264324_Identification_of_Periodontal Pathogens_in_Atheromatous_Plaques

[8] Kozarov EV, Dorn BR, Shelburne CE, Dunn WA, Progulske-Fox A. Human atherosclerotic plaque contains viable invasive Actinobacillus actinomycetemcomitans and Porphyromonas gingivalis. Arteriosclerosis, Thrombosis, and Vascular Biology. 2005;25:e17.

https://www.ahajournals.org/doi/full/10.1161/01.ATV.0000155018.67835.1a?url_v er=Z39.88-2003\&rfr_id=ori\%3Arid\%3Acrossref.org\&rfr_dat=cr_pub\%3Dpubmed

[9] Stelzel M, Conrads G, Pankuweit S, Maisch B, Vogt S, Moosdorf R, Flores-deJacoby L. Detection of Porphyromonas gingivalis DNA in aortic tissue by PCR. J Periodontol. 2002;73(8):868-70. https://aap.onlinelibrary.wiley.com/doi/pdf/10.1902/jop.2002.73.8.868

[10] Giacona MB, Papapanou PN, Lamster IB, Rong LL, Agati VD, Schmidt AM, at al. Porphyromonas gingivalis induces its uptake by human macrophages and promotes foam cell formation in vitro. FEMS Microbiology Letters 241. 2004;95101. https://onlinelibrary.wiley.com/doi/pdf/10.1016/j.femsle.2004.10.009

[11] Li XY, Wang C, Xiang XR, Chen FC, Yang CM, Wu J. Porphyromonas gingivalis lipopolysaccharide increases lipid accumulation by affecting CD36 and ATPbinding cassette transporter A1 in macrophages. Oncology Report. 2013;30:13291336. https://www.spandidos-publications.com/or/30/3/1329

[12] Gani DK, Lakshmi D, Krishnan R, Emmadi P. Evaluation of C-reactive protein and interleukin- 6 in the peripheral blood of patients with chronic periodontitis. $\mathrm{J}$ Indian Soc Periodontol. 2009;13(2):69-74. https://www.ncbi.nlm.nih.gov/pmc/articles/PMC2847127/

[13] Brodala N, Merricks EP, Bellinger DA, Damrongsri D, Offenbacher S, Beck J, et al. Porphyromonas gingivalis bacteremia induces coronary and aortic atherosclerotic in normocholesterolemic and hypercholesterolemic pigs. Journal American Heart Association. 2005:1446-51. https://www.ahajournals.org/doi/full/10.1161/01.ATV.0000167525.69400.9c

[14] Abe TA, Hajishengallis G. Optimization of the ligature-induced periodontitis model in mice. J Immunol Methods. 2013;394(1-2):49-54.

https://www.sciencedirect.com/science/article/pii/S002217591300149X?via\%3Dih $\underline{\mathrm{ub}}$

[15] Fava C, Montagnana M. Atherosclerosis is an inflammatory disease which lacks a common anti-inflammatory therapy: How human genetics can help to this issue. A Narrative Review. Front Pharmacol. 2018;9:55.

https://www.frontiersin.org/articles/10.3389/fphar.2018.00055/full

[16] Spahr S, Klein E, Khuseyinova N, Boeckh C, Muche R, Kunze M, et al. Periodontal infections and coronary heart disease: Role of periodontal bacteria and importance of total pathogen burden in the coronary event and periodontal disease (CORODONT) Study. Arch Intern Med. 2006;166:554-9. https://jamanetwork.com/journals/jamainternalmedicine/fullarticle/409905 
Susilawati et al. (2020): Coronary artery disease in rat model. February 2020. 23(3A)

[17] Tuttolomondo A, Raimondo DD, Pecoraro R, Arnao V, Pinto A, Licata G. Atherosclerosis as an inflammatory disease. Current Pharmaceutical Design. 2012;18(28):4266-88.

https://www.ingentaconnect.com/content/ben/cpd/2012/00000018/00000028/art000 $\underline{02}$

[18] Mougeot JLC, Stevens CB, Paster BJ, Brennan MT, Lockhart PB, Mougeot FKB. Porphyromonas gingivalis is the most abundant species detected in coronary and femoral arteries. J Oral Microbiol. 2017;9(1):1-9.

https://www.tandfonline.com/doi/abs/10.1080/20002297.2017.1281562 